\title{
Frequency-Dependent Smith-Chart Model as Applied to Integrated Circuit Package Antenna Design
}

\author{
Settapong Malisuwan and Jesada Sivaraks
}

\begin{abstract}
In this paper, the frequency-dependent Smith-Chart representation is utilized to improve the accuracy of integrated circuit package antenna (ICPA) design. The purpose of this research is to reveal that the frequency-dependent Smith-Chart method (FDSC) is more exact than alternative models adopted in past research. The results in this research show that the FDSC model is more accurate than the previous model in the literature by comparing to the measure results. The method utilized in this paper can be applied to use in CAD applications with fast and user-friendly implementations.
\end{abstract} CAD.

Index Terms-Frequency dependent, Smith-chart, ICPA,

\section{INTRODUCTION}

The Smith-chart is an exceptional graphical tool used in high-frequency engineering to design amplifiers, filters, circuits and other impedance and reflection coefficient charts. Although there are other tools adopted to design components aforementioned, the Smith-chart is vastly known and is the most popular tool amongst them. It is a significant tool used in modern computer-aided design software (CAD) for high frequency designs.

The frequency-dependent Smith chart (FDSC) was proposed in [1], [2]. The results in [1], [2] suggests that FDSC gives more accurate results than the models adopted in past literature [3]. Basically in FDSC, the frequency-dependent characteristic impedance is addressed in higher detail in included in the algorithm hence risk of possible error is reduced. The purpose of this paper is to present the accuracy of FDSC model to design the ICPA.

In the next sections, the FDSC representation is explained and utilized to design the microstrip patch antenna and ICPA circuit.

\section{FREQUENCY-DEPENDENT SMITH-CHART REPRESENTATION}

In this section, we describe the concept of FDSC model prior to apply the concept to design the ICPA circuit to achieve the objective.

The concept of microstrip-based Cole-Cole diagram is adopted to create a frequency-dependent (lossy) Smith-chart

Manuscript received April 11, 2013; revised May 22, 2013. This work was supported in full by National Broadcasting and Telecommunications Commission of Thailand (NBTC).

The authors are with the National Broadcasting and Telecommunications Commission (NBTC), Bangkok, Thailand (email: settapong.m@nbtc.go.th, jesada.s@nbtc.go.th) to scrutinize microstrip line characteristics [1]. Prior to analyzing the frequency-dependent Smith-chart variables, the capacitance parameter in microstrip-line system should be analyzed. The capacitance per unit length of the classical parallel-plate capacitor is [4]:

$$
C=\varepsilon \frac{w}{h}
$$

A simple frequency-dependent capacitance of the parallel-plate capacitor can be expressed in any frequency-dependent attributes of $\varepsilon$ which is

$$
C(\omega)=\varepsilon_{0} \varepsilon^{*}(\omega) \frac{w}{h}
$$

where $\varepsilon^{*}(\omega)$ is a complex permittivity is expressed as $\varepsilon^{\prime}(\omega)-j \varepsilon^{\prime \prime}(\omega)$.

Therefore,

$$
C(\omega)=\varepsilon_{0} \varepsilon^{\prime}(\omega) \frac{w}{h}-j \varepsilon_{0} \varepsilon^{\prime \prime}(\omega) \frac{w}{h}
$$

Referring to the equivalent Cole-Cole diagram deduced for a parallel-plate microstrip line in [3] is substitute into Eqn. (3). Hence,

$$
\begin{aligned}
& C(\omega)=C\left(\frac{1}{1+Q(\omega)}\left[Q(\omega)+\frac{\varepsilon_{e f f}(0)}{\varepsilon_{r}}\right]\right) \\
& -j \frac{C}{\varepsilon_{r}}\left[\varepsilon_{u}^{\prime \prime}(\omega)+\varepsilon_{c}^{\prime \prime}(\omega)+\varepsilon_{d}^{\prime \prime}(\omega)\right]
\end{aligned}
$$

where $C=\varepsilon_{0} \varepsilon_{r}(w / h)$.

For simplicity, the coefficients of Eqn. (4) are defined as follows:

$$
\begin{aligned}
& A(\omega)=\frac{1}{1+Q(\omega)}\left[Q(\omega)+\frac{\varepsilon_{e f f}(0)}{\varepsilon_{r}}\right] \\
& B(\omega)=\frac{1}{\varepsilon_{r}}\left[\varepsilon_{u}^{\prime \prime}(\omega)+\varepsilon_{c}^{\prime \prime}(\omega)+\varepsilon_{d}^{\prime \prime}(\omega)\right]
\end{aligned}
$$

In general, the characteristic impedance of a transmission line is given by: 


$$
Z_{0}=\sqrt{\frac{R+j \omega L}{G+j \omega C}}
$$

where $R, L, G, C$ are per unit length quantities defined as follows:

$R=$ resistance per unit length in $\Omega / \mathrm{m}$.

$L=$ inductance per unit length in $\mathrm{H} / \mathrm{m}$.

$G=$ conductance per unit length in $\mathrm{S} / \mathrm{m}$.

$C=$ capacitance per unit length in $\mathrm{F} / \mathrm{m}$. [1]

If $\mathrm{G}$ and $\mathrm{C}$ are neglected, the characteristic impedance can be written as:

$$
Z_{0}=\sqrt{\frac{L}{C}}
$$

To achieve frequency-dependent characteristic impedance $\left(Z_{0}{ }^{\prime}(\omega)\right)$, the frequency-dependent capacitance $(C(\omega))$ of Eqn. (4) is replaced into the capacitance (C) in Eqn. (8).Therefore, frequency-dependent characteristic impedance is

$$
Z_{0}^{\prime}(\omega)=\sqrt{\frac{L}{C[A(\omega)-j B(\omega)]}}=\frac{Z_{0}}{\sqrt{A(\omega)-j B(\omega)}}
$$

Now, the frequency-dependent (lossy) Smith-chart can be derived through input of $Z_{0}^{\prime}(\omega)$ in Eqn. (9) into the normalized terminal impedance expression as done in traditional Smith-chart model [5]. Therefore the normalized terminal impedance $Z_{L}^{\prime}$ is

$$
Z_{L}^{\prime}=\frac{Z_{L}}{Z_{0}^{\prime}(\omega)}=b r+j b x \quad(\text { Dimensionless })
$$

As $r$ and $x$ are the normalized resistance and normalized reactance, and $b=\sqrt{A(\omega)-j b(\omega)}$.

The voltage reflection coefficient of present Smith chart is

$$
\Gamma^{\prime}=\Gamma_{r}^{\prime}+j \Gamma_{i}^{\prime}=\frac{Z_{L}^{\prime}-1}{Z_{L}^{\prime}+1}
$$

or

$$
Z_{L}^{\prime}=\frac{Z_{L}}{Z_{0}^{\prime}(\omega)}=b r+j b x=\frac{\left(1+\Gamma_{r}^{\prime}\right)+j \Gamma_{i}^{\prime}}{\left(1-\Gamma_{r}^{\prime}\right)-j \Gamma_{i}^{\prime}}
$$

Now, the set of equations representing the modified Smith-chart is expressed as:

$$
\left(\Gamma_{r}^{\prime}-\frac{b r}{1+b r}\right)^{2}+\Gamma_{i}^{\prime 2}=\frac{1}{(1+b r)^{2}}
$$

and

$$
\left(\Gamma_{r}^{\prime}-1\right)^{2}+\left(\Gamma_{i}^{\prime}-\frac{1}{b x}\right)^{2}=\left(\frac{1}{b x}\right)^{2}
$$

As mentioned earlier in this paper, the objective of this research is to utilize the FDSC model to design the ICPA. To present the accuracy of the FDSC model, this paper uses the characteristics of the ICPA circuit from [6] to construct the FDSC diagram to meet this specific case [6].

The FDSC diagram is compared with a standard Smith-Chart in Fig. 1.

It can be seen that when the lossy characteristics (substrate loss, conductor loss, and frequency-dependent characteristic impedance of the microstrip line) are included in the calculation, the Smith-chart takes the form of a spiral. As well known in lossy transmission line theory that, when attenuation as a function of line-length is plotted on the Smith chart, it also takes the form of a spiral.

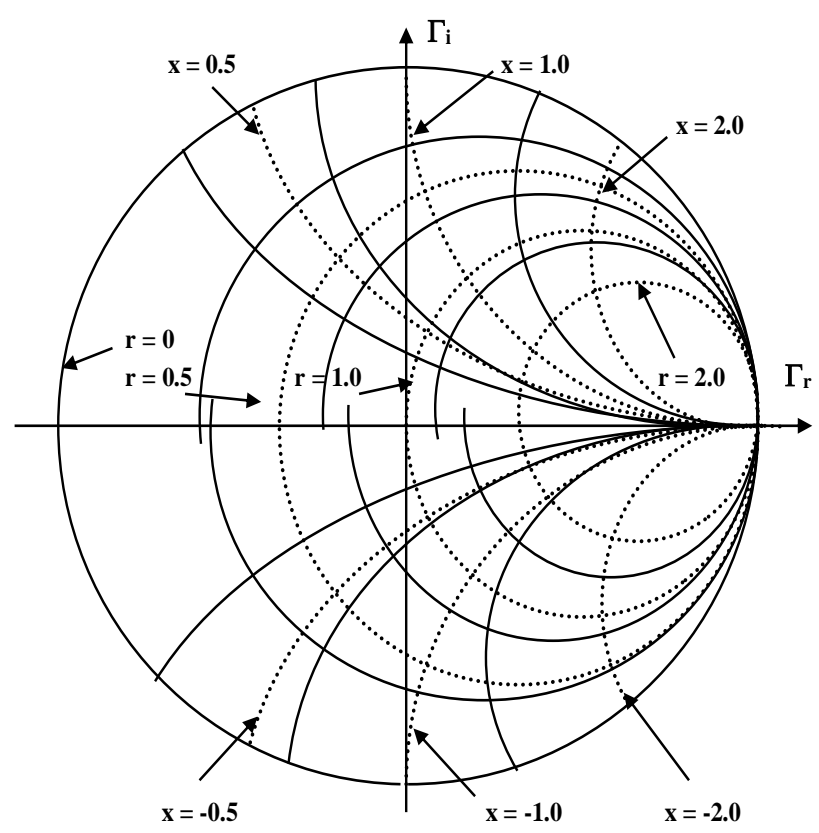

Fig. 1. The frequency-dependent (lossy) Smith chart with dielectric constant $=5.9$ and the size of the microstripstructure from [6] FDSC Standard Smith chart

\section{ICPA AND ITS CIRCUIT MODEL}

"Any printed circuit antenna can be used for the ICPA" [3]. The microstrip patch antenna is narrowband and widebeam. Its advantage is that it is relatively inexpensive to manufacture and design especially on UHF band and higher. Consequently, ICPA thermal performance can be augmented by the microstrip patch. Its characteristic is simply modeled as a parallel resonant RLC circuit. To evaluate its characteristics, the calculation based on RLC circuit is most often used.

In this paper, we used the ICPA in the custom designed package format from the previous research as shown in Fig. 2 

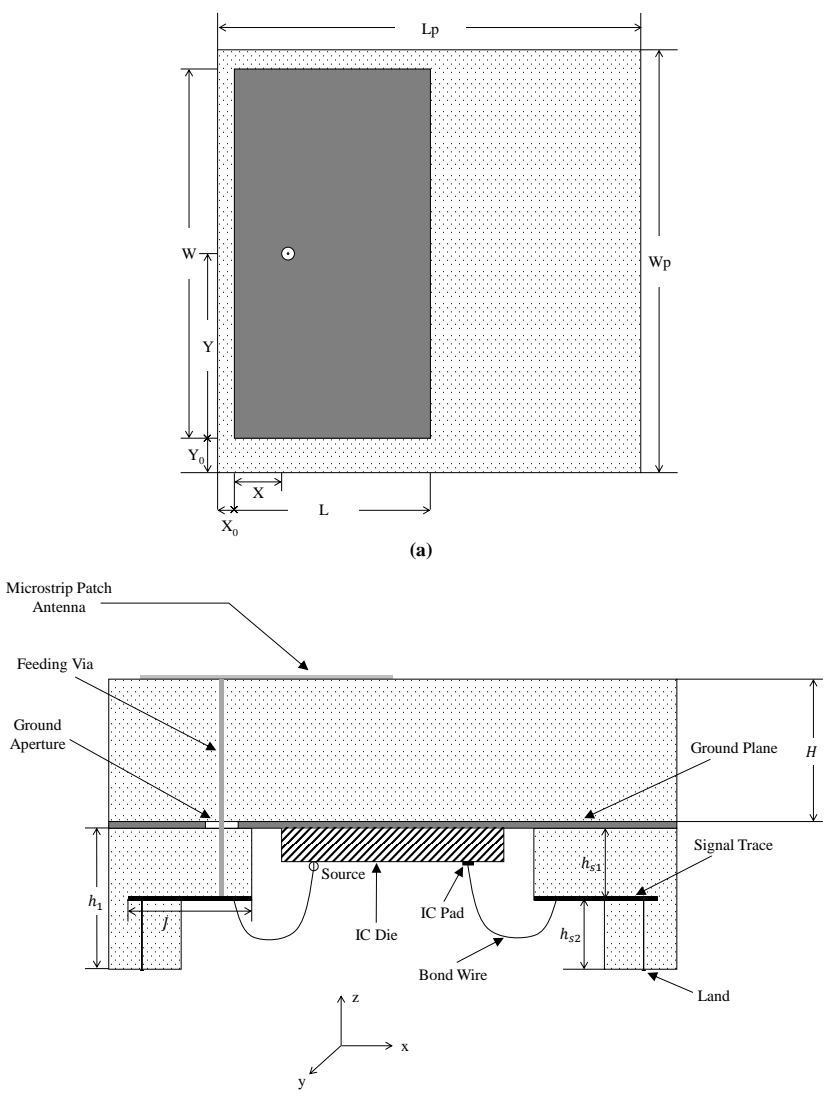

(b)

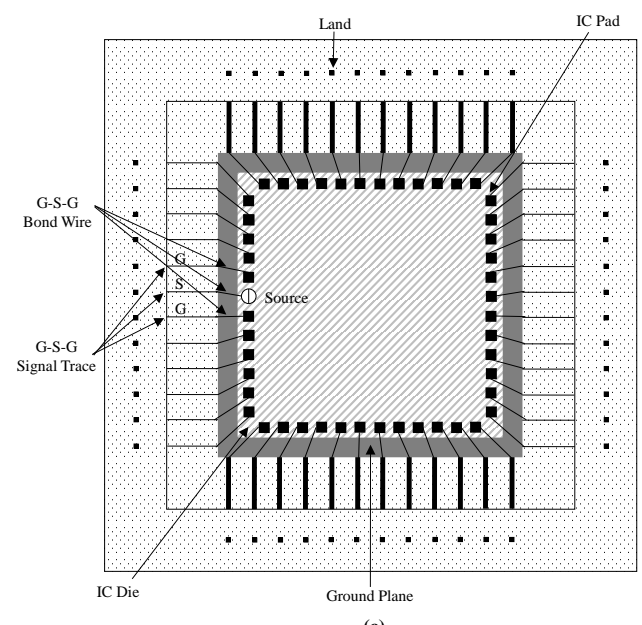

(c)

Fig. 2. ICPA: (a) Top view, (b) cross-section view, and (c) bottom view. (Fig. 2 is from [6])

From Fig. 2, Signal Trace from the carried chip feed the signal to the microstrip patch antenna of the ICPA in the formation of G-S-G bond wires. The corresponding circuit model of the ICPA feeding network is illustrated in Fig. 3. The equivalent RLC can be represented the 5 composites of the ICPA feeding network: the section of microstrip antenna: the section of feeding via underground plane, the section of GSG signal Traces, the section of GSG bond wires, and the section of vias to lands and lands.Next step is the calculation of RLC value in the circuit model of the microstrip patch anetenna. The feeding via that is above the ground plane corresponds with inductive reactance term as shown below [2], [3]

$$
X L=\frac{377 f_{r} H}{C_{0}} \ln \left(\frac{C_{0}}{\pi f_{r} d_{v} \sqrt{\varepsilon_{r}}}\right)
$$

\section{Therefore}

$C_{0}$ represents velocity of light,

$d_{v}$ is diameter of the feeding via,

$H$ represents thickness of the substrate between the microstrip patch and ground plane,

$\varepsilon_{r}$ is the relative permittivity of the substrate, and

$f_{r}$ is the resonant frequency of the microstrip patch antenna [3].

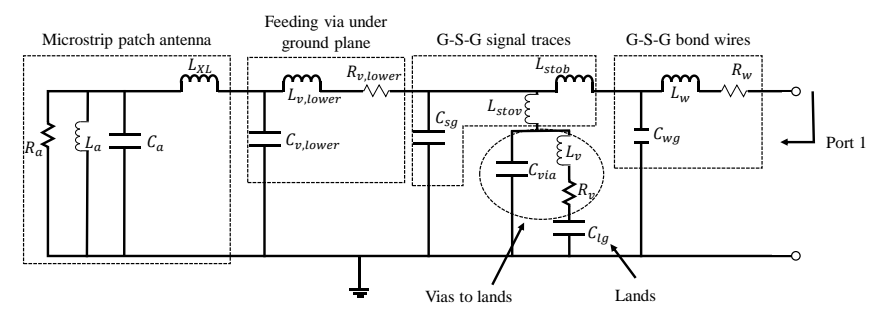

Fig. 3. Equivalent circuit model of the ICPA feeding network. (Fig. 3 is from [6])

The resonant resistance $R_{a}$ of the parallel $R L C$ circuit is [3]:

$$
R_{a}=\frac{Q_{\text {total }} H}{\pi f_{r} \varepsilon_{d y n} \varepsilon_{0} W L_{e f f}} \cos 2\left(\frac{\pi X_{e f f}}{L_{e f f}}\right)
$$

So, length $L_{e f f}$ takes into account the influence of the fringing field at the corners and the dielectric in homogeneity of the ICPA; as a result, the distance from the feeding point to the patch edge $X$ substituted with

$$
X_{e f f}=X+\frac{\left(L_{e f f}-L\right)}{2}
$$

Eq. (16) $\varepsilon_{d y n}$ signifies the dynamic permittivity, defined as "function of the dimensions of the ICPA and relative permittivity $\varepsilon_{r}$ as well as the different modes field distribution"[6]. Calculations of $\varepsilon_{d y n}, C_{e 1, \text { stat }}(\varepsilon)$ characterize border capacitance on one side of the patch length whereas, $L$ and $C_{e 2 \text {,stat }}(\varepsilon)$ stands for capacitance on one side of a patch with width $W$. More information can be found in [6].

"Therefore, impedance of the dielectric filled microstrip patch of width $W$ "is [3]

$$
Z a(W)=\frac{\sqrt{\varepsilon_{e f f}(W)}}{c_{0} C_{t w}\left(W, H, \varepsilon_{r}\right)}
$$

and

$$
P_{a}(W)=\frac{2 \pi\left[\frac{W}{H}+\frac{\frac{W}{(\pi H)}}{\frac{W}{(2 H)}+0.94}\right]\left[1+\frac{H}{W}\right]}{\left\{\frac{W}{H}+\frac{2}{\pi} \ln \left[2 \pi e\left(\frac{W}{2 H}+0.94\right)\right]\right\}^{2}}
$$

Aforementioned, $f_{r}$ is the resonant frequency of the microstrip patch antenna where the actual part of the input impedance achieves maximum, the additive reactance, that is $X L$ will not alter value of the resonant frequency 


$$
f_{r}=\frac{c_{0}}{2 \sqrt{\varepsilon_{d y n}}} \sqrt{\left(\frac{m}{W_{e f f}}\right)^{2}+\left(\frac{n}{L_{e f f}}\right)^{2}}
$$$$
L_{w}=2 l_{w} \ln \left(\frac{4 h_{w}}{d_{w}}\right) n H
$$

The effective width $W_{\text {eff }}$ and length $L_{e f f}$ is from [6]. "For the circuit model of feeding via under ground plane, $L_{v}$,lower and $R_{v, l o w e r}$ are the inductance and resistance of the feeding via under ground plane"[3]. The inductance can be derived using the following equation:

$$
L_{v, \text { lower }}=20 l_{v, \text { lower }}\left[\ln \left(\frac{2 l_{v, \text { lower }}}{r_{v, \text { lower }}}\right)-1\right] n H
$$

where $l_{v, \text { lower }}, r_{v, \text { lower }}$ are the length and the radius of the feeding via (in millimeters) under the ground plane

$$
R_{v, \text { lower }}=\frac{l_{v, \text { lower }}}{\sigma S_{v, \text { lower }}}
$$

"So, $l_{v, \text { lower }}, S_{v \text {,lower }}$ and $\sigma$ are the length, the cross-section area, and the conductivity of the feeding via under the ground plane respectively"[6].

$C_{v \text {,lower }}$ "is the capacitance amidst the feeding via under the ground plane and the ground plane also the shorting vias. It can be derived by the method of moments"[6].

$C_{s g}$ signifies the capacitance of the signal traces and is a CPW structure. In this study it is derived by the conformal mapping method [6] For circuit model of G-S-G signal traces [6]

$$
C_{s g}=2 \varepsilon_{0} \varepsilon_{s, \text { eff }}\left[\frac{K\left(k_{1}\right)}{K\left(k_{1}^{\prime}\right)}+\frac{K\left(k_{0}\right)}{K\left(k_{0}^{\prime}\right)}\right] \times l_{8}
$$

where $K\left(k_{i}\right)$ and $\varepsilon_{s, \text { eff }}$ can be found in [6].

The signal trace inductance is derived by [6]

$$
L_{s g}=\frac{\mu_{0} l_{s}}{2 \pi}\left\{\begin{array}{l}
\operatorname{arsinh}\left(\frac{l_{s}}{w_{s}+t}\right)+\frac{l_{s}}{w_{s}+t} \\
\operatorname{arsinh}\left(\frac{w_{s}+t}{l_{s}}\right) \\
+\frac{w_{s}+t}{3 l_{s}}-\frac{1}{3}\left(\frac{l_{s}}{w_{s}+t}\right)^{2} \\
{\left[\begin{array}{l}
\left(\frac{1+\left(w_{s}+t\right)^{2}}{l_{s}^{2}}\right)^{\frac{3}{2}}-1
\end{array}\right]}
\end{array}\right\}
$$

Hence, $w_{s}$ signifies the "width of the signal trace", $t$ is the "thickness of signal trace," $l_{s}$ signifies "length of the signal trace"[6]. In Fig. 3, $L_{\text {stob }}$ and $L_{\text {stov }}$ represents "inductances of the signal trace," which is the length of signal trace to bond wire and signal trace to the via linking the land.

For circuit model of G-S-G bond wires, the inductance and capacitance of the bond wire is [7]:

$$
C_{w}=\frac{0.5563 l_{w}}{\ln \left(\frac{4 h_{w}}{d_{w}}\right)} p F
$$

where $l_{w}, d_{w}$ and $h_{w}$ are in cm, $l_{w}, d_{w}$ and $h_{w}$ are the length of the bond wire, the diameter of the bond wire, and the distance from the bond wire to the ground plane [6].

\section{RESUlTS AND DisCUSSIONS}

The modeled and FDSC results are analyzed and a measurement for ICPA is stated from [6]. Referring to the research in [6], the "ICPA designed with Ferro-A6 LTCC material system with dielectric constant of 5.9 and loss tangent of 0.002 at $6 \mathrm{GHz}^{\prime}$ [6].

The fabricate part of microstrip patch from ICPA which taken from [6]. The ICPA measures $17 \times 17 \times 1.6 \mathrm{~mm}$. The top, middle and bottom layers have thickness of $.8,0.4$, and $0.4 \mathrm{~mm}$, correspondingly. The vias have diameter of $100 \mathrm{~m}$ whereas the traces is the size of $2 \times 0.4 \mathrm{~mm}$. The lands are squared with length measured as $0.34 \mathrm{~mm}$. The feeding is 1.2 $\mathrm{mm}$ in length whereas vias to the ground plan and lands are $0.4 \mathrm{~mm}$ in length. The gap diameter for the feeding via to go through is $0.6 \mathrm{~mm}$. The feeding and grounded bond wires have diameter of $32.5 \mathrm{~m}$ and are $0.22 \mathrm{~mm}$ in length. Illustrated in Fig 1, the dimensions of the microstrip patch antenna are $L=9.9 \mathrm{~mm}, W=15 \mathrm{~mm}, X_{0}=0.9 \mathrm{~mm}, Y_{0} 1.4 \mathrm{~mm}, X=2.35 \mathrm{~mm}$, and $Y=7.5 \mathrm{~mm}$. [6]

By applying the FDSC model, the measured and modeled results from are mapped on the frequency-dependent Smith-Chart model in Fig. 1 and then compared with the previous results in [6].

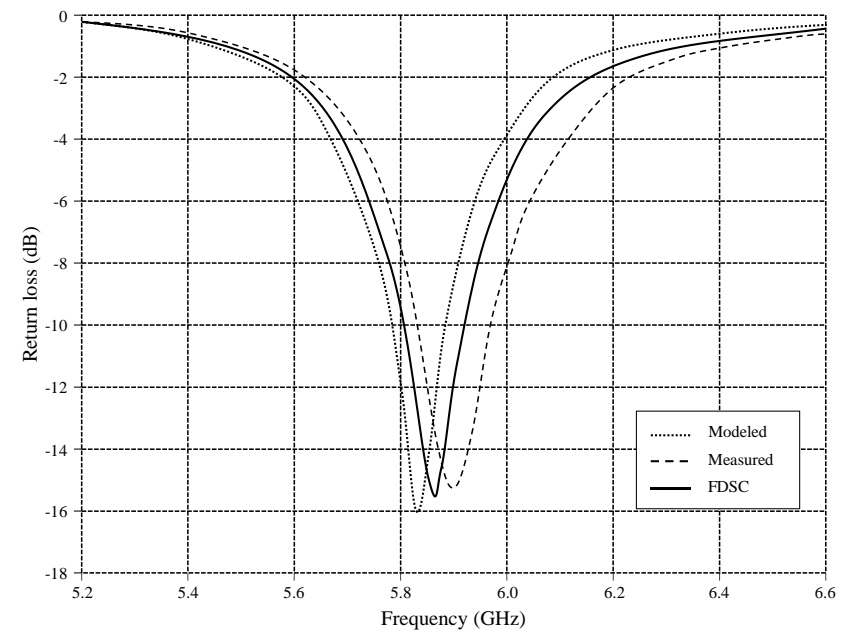

Fig. 4. Comparison of the return loss for the ICPA

Fig. 4 illustrates the model and measurements of return loss from for the microstrip Patch Antenna in comparison with the FDSC result.

As shown in Fig. 4 the center frequencies of the impedance bandwidth are 5.9, 5.83 and 5.86GHz for the measured [6], modeled, and the FDSC results, respectively. The difference 
between the measuresments and model is $0.07 \mathrm{GHz}(0.07 / 5.9$ $=1.19 \%)$, while the difference between the measurements and the FDSC method is only $0.04 \mathrm{GHz}(0.04 / 5.9=0.68 \%)$ as shown in TABLE.

TABLE I: COMPARISON OF THE RETURN LOSS FOR THE ICPA

\begin{tabular}{|c|c|c|}
\hline & Frequency $(\mathrm{GHz})$ & $\Delta \%$ \\
\hline Measured [6] & 5.90 & - \\
\hline Modeled[6] & 5.83 & 1.19 \\
\hline FDSC & 5.86 & 0.68 \\
\hline
\end{tabular}

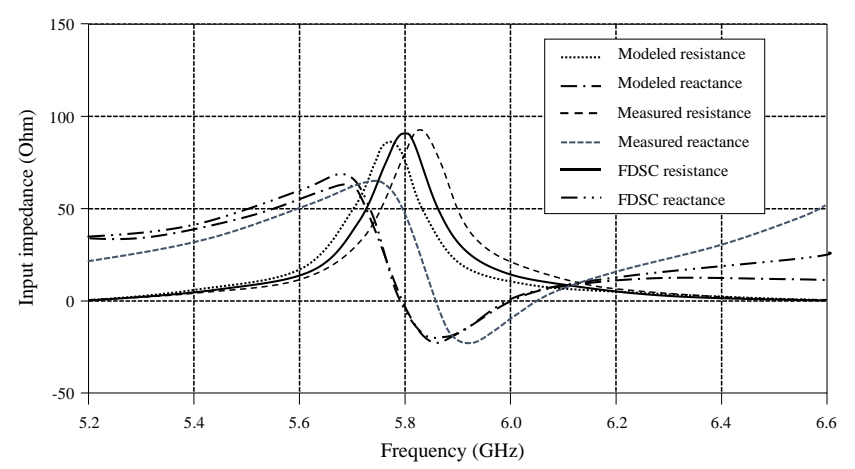

Fig. 5. Comparison of the input impedance for the ICPA.

Fig. 5 shows the measurement and the FDSC input impedance [6]. The resonant frequencies are 5.83, 5.77 and $5.80 \mathrm{GHz}$ from the measured model and FDSC results, respectively. The difference between the measured and the modeled [6] is $0.06 \mathrm{GHz}(0.06 / 5.83=1.03 \%)$, while the difference between the measured and FDSC model is only $0.03 \mathrm{GHz}(0.03 / 5.83=0.51 \%)$ as shown in TABLE II:

TABLE II: RESONANT FREQUENCY

\begin{tabular}{|c|c|c|}
\hline & Frequency $(\mathrm{GHz})$ & $\Delta \%$ \\
\hline Measured [6] & 5.83 & - \\
\hline Modeled [6] & 5.77 & 1.03 \\
\hline FDSC & 5.80 & 0.51 \\
\hline
\end{tabular}

The resonant resistance of the $R L C$ parallel circuit is $89.3 \Omega, 85.45 \Omega$, and $87.40 \Omega$ from the measured, modeled, and FDSC results, respectively. The difference between the measured and modeled is $3.85 \Omega(3.85 / 87.3=4.3 \%)$, while the difference between the measured and FDSC is only $1.9 \Omega$ $(1.9 / 89.3=2.1 \%)$ as shown in TABLE.

TABLE III: RESONANT RESISTANCE
\begin{tabular}{|c|c|c|}
\hline & $\operatorname{Ohm}(\Omega)$ & $\Delta \%$ \\
\hline Measured [6] & 89.30 & - \\
\hline Modeled [6] & 85.45 & 4.3 \\
\hline FDSC & 87.40 & 2.1 \\
\hline
\end{tabular}

\section{CONCLUDING REMARKS}

The use of the frequency-dependent Smith-Chart model is proved to be an accurate method to calculate and model the the frequency-dependent characteristics of microstrip antennas and ICPA applications.

This paper demonstrated a user-friendly and accurate method on verifying existing formulas and measurements.

Overall, the method explained in this research recommends an effective strategy for portraying the frequency-dependent characteristic of microstrip antennas and ICPA circuit via the FDSC model.

The RF engineers can utilize the FDSC model in this paper to facilitate computer-aided design (CAD) software.

\section{REFERENCES}

[1] S. Malisuwan, M. Charoenwattanaporn, U. Goenchanart, and V. Ungvichian, "Microstrip Antenna for wireless LAN Applications by applying Modified Smith-Chart Representation," International Journal of Computer, Internet and Management, vol.11, no. 3, 2003

[2] S. Malisuwan, P. S. Neelakanta, and V. Ungvichian, "A Cole-Cole diagram representation of microstrip structure," Journal of the Applied Computational Electromagnetics Society, vol.15, no. 3, 2000.

[3] F. Abboud, J. P. Damiano, and A. Papiernik, "Simple model for the inputimpedance of coax-fed rectangular microstrip patch antenna for CAD," in Proc. Inst. Elect. Eng. Microwaves, Antennas and Propagation, vol. 135, no. 5, pp. 323-326, Oct. 1988.

[4] C. S. Walker, Capacitance, Inductance and Crosstalk Analysis. Norwood, MA: Artech House, 1990.

[5] J. C. Freeman, Fundamentals of Microwave Transmission Lines, John Wiley \& Sons, Inc., New York, 1995.

[6] J. J. Wang, Y. P. Zhang, K. M. Chua, and A. C. W. Lu, "Circuit Model of Microstrip Patch Antenna on Ceramic Land Grid Array Package for Antenna-Chip Co design of Highly Integrated RF Transceivers," IEEE Tran. on Antennas and Propagation, vol. 53, no.12, Dec, 2005.

[7] E. Pettenpaul, H. Kapusta, A. Weisgerber, H. Mampe, J. Luginsland, and I. Wolff, "CAD models of lumped elements on GaAs up to 18 GHz," IEEE Trans. Microw. Theory Tech., vol. 36, no. 2, pp. 294-304, Feb. 1988.

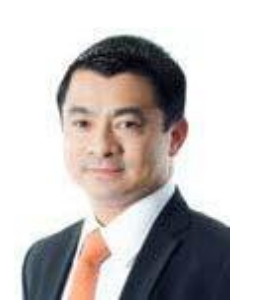

Settapong Malisuwan was born on $24^{\text {th }}$ March 1966 in Bangkok, Thailand. He received his $\mathrm{PhD}$ in electrical engineering (telecommunications), specializing in mobile communication systems from Florida Atlantic University (State University System of Florida), Boca Raton in 2000. He received an MSc in electrical engineering in mobile communications system, from George Washington University in 1996, an MSc in electrical engineering in telecommunication engineering from Georgia Institute of Technology in 1992 and a BSc in electrical engineering from the Chulachomklao Royal Military Academy, Nakhon-Nayok, Thailand in 1990. He served in the Royal Thai Armed Forces for more than 25 years and is currently the Vice Chairman of National Broadcasting and Telecommunications, Bangkok, Thailand. His research interests are in efficient spectrum management and Telecommunications policy and management in Thailand. Col. Dr. Settapong Malisuwan is currently the Elected Vice Chairman and Board Member in the National Broadcasting and Telecommunications Commission, Thailand.

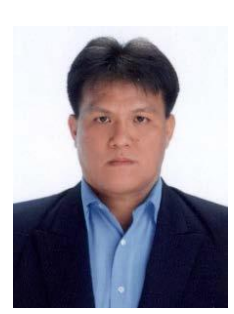

Jesada Sivaraks was born on $12^{\text {th }}$ May 1970 in Bangkok, Thailand. He received his MSEE degree from Oklahoma State University in 1996 and BEng from King Mongkut"s Institute of Technology, Thailand. He completed his $\mathrm{PhD}$ in electrical engineering at Florida Atlantic University, Boca Raton, FL in 2001. Since 2011, he has been working in National Broadcasting and Telecommunications Commission as the Secretary to the Vice Chairman. HisPhD work is on the system aspects of Bluetooth, WLAN and Mobile IP/CDPD. His current research interests are in telecommunication planning and related system analysis and efficient spectrum management. He is a member of Tau Beta Pi, Florida Epsilon and was an Honorary Advisory's Chairman of Science \& Technology committee of Parliament in 2009. 